\title{
Observation of the time dependence of $B_{d}^{0}-\bar{B}_{d}^{0}$ mixing
}

\author{
The ALEPH Collaboration
}

\begin{abstract}
The time dependence of $B_{d}^{0}-\bar{B}_{d}^{0}$ oscillations has been observed using events with a $D^{*}$, decaying into a $D^{0} \pi^{+}$, and a lepton in opposite hemispheres. The time dependence of the oscillations is derived from the displacement of the $D^{0}$ vertex and the $D^{*}$-lepton charge correlation. From a fit for the oscillation frequency the mass difference of the $B_{d}^{0}$ states is measured:

$$
\Delta m=\left[3.44{ }_{-0.70}^{+0.65}(\text { stat }){ }_{-0.20}^{+0.26}(\text { sys })\right] 10^{-4} \mathrm{eV} / \mathrm{c}^{2} .
$$
\end{abstract}

To be submitted to Physics Letters B 


\section{Introduction}

Mixing in the $B^{0} \bar{B}^{0}$ system is formally described in the same way as the well known $K^{0} \bar{K}^{0}$ system. For the present purpose the (small) effects of CP violation can be neglected, so that the states of well defined flavour, $B^{0}$ and $\bar{B}^{0}$, in terms of the states with well defined mass and lifetime, $B_{1}$ and $B_{2}$, are:

$$
B^{0}=\frac{B_{1}+B_{2}}{\sqrt{2}} \quad \bar{B}^{0}=\frac{B_{1}-B_{2}}{\sqrt{2}} .
$$

In contrast to the kaon system, for the B meson the decay width difference is expected to be small [1]; in the following it is neglected. The probability that a meson created as a $B^{0}$ meson will be a $B^{0}$ meson at time $t$ is then:

$$
P(t)=\frac{1}{2} \exp (-\Gamma t / \hbar)[1+\cos (\Delta m t / \hbar)],
$$

and the probability that it will be a $\bar{B}^{0}$ at time $t$ is:

$$
P(t)=\frac{1}{2} \exp (-\Gamma t / \hbar)[1-\cos (\Delta m t / \hbar)],
$$

where $\Gamma$ is the decay width, and $\Delta m$ is the difference of the two mass eigenstates. The total probability $\chi$ that a $B^{0}$ decays as a $\bar{B}^{0}$ is then:

$$
\chi=\frac{1}{2} \frac{\left(\frac{\Delta m}{\Gamma}\right)^{2}}{1+\left(\frac{\Delta m}{\Gamma}\right)^{2}}
$$

The first evidence for $B^{0}-\bar{B}^{0}$ mixing was found by UA1 [2]. However, this measurement could not distinguish between $B_{d}^{0}$ and $B_{s}^{0}$. This experiment measured an average mixing parameter $\hat{\chi}=f_{s} \chi_{s}+f_{d} \chi_{d}$, where $\chi_{i}$ are the mixing parameters of the respective mesons and $f_{i}$ their relative abundance. $B_{d}^{0}$ mixing was established by ARGUS [3] and confirmed by CLEO [4]. Other measurements of mixing followed later especially at LEP [5], with more precise results on $\hat{\chi}$. However, all these experiments could only measure the time integrated quantity $\chi$. The characteristic time dependence has not previously been observed. While measurements of $B_{s}^{0}$ oscillations need high statistics and a very good resolution of the $B_{s}^{0}$ decay time, the requirements to resolve $B_{d}^{0}$ mixing, which should oscillate more slowly, are easier to meet. Combining the results of ARGUS and CLEO the mixing parameter $\chi_{d}$ is $0.159 \pm 0.025$ which corresponds to $\frac{\Delta m}{\Gamma}=0.68 \pm 0.10$. Hence, a complete oscillation period of the $B_{d}^{0}$ takes about 9 lifetimes. The $B_{d}^{0}$ has equal probability to decay as a $B_{d}^{0}$ or $\bar{B}_{d}^{0}$ at about 2.2 lifetimes. At LEP energies this corresponds to a flight path of $6 \mathrm{~mm}$.

\section{$2 \quad D^{*}$-lepton correlations}

In order to measure the oscillation frequency $\Delta m$ one has to tag the state of the $B_{d}^{0}$ at the time of production and at the time of decay. The state of the $B_{d}^{0}$ at production time can be tagged via a semileptonic decay of the $\mathrm{B}$ in the opposite jet. The state at decay time can be tagged by a $D^{* \pm}$ :

$$
B_{d}^{0} \rightarrow D^{*-} X ; \bar{B}_{d}^{0} \rightarrow D^{*+} X .
$$


A $D^{*-} l^{-}$pair is called like sign and tags an unmixed event. A $D^{*-} l^{+}$pair is called unlike sign and tags a mixed event. The charge correlation function $C_{Q}(t)$ is defined as:

$$
C_{Q}(t) \equiv \frac{N^{\text {like }}(t)-N^{\text {unlike }}(t)}{N^{\text {like }}(t)+N^{\text {unlike }}(t)}=\frac{N^{\text {unmixed }}(t)-N^{\text {mixed }}(t)}{N^{\text {unmixed }}(t)+N^{\text {mixed }}(t)}
$$

Of course this equation holds only in the case of perfect charge tagging and no background. This quantity would then oscillate between +1 and -1 with the angular frequency $\Delta m$. However, in practice this behaviour is modified:

The $B_{d}^{0}$ momentum is not reconstructed and the proper decay time cannot be calculated from the decay length. Hence the analysis is performed with the decay length instead, the oscillation frequency transforming into $\Delta m / \hbar c \beta \gamma$ (where $\beta \gamma$ is the Lorentz boost of the $\left.B_{d}^{0}\right)$.

The $D^{*}$ vertex cannot be reconstructed with appropriate precision because the slow pion from the $D^{*} \rightarrow D^{0} \pi^{+}$is almost parallel with the $D^{0}$. Hence the analysis is performed with the sum of the $B_{d}^{0}$ and $D^{0}$ decay length as parameter. The effect of the additional $D^{0}$ flight path has to be taken into account and the decay length distribution has also to be convoluted with the $B_{d}^{0}$ and $D^{0}$ momentum spectra.

In some fraction of the events the lepton or $D^{*}$ does not tag the correct B-state. The lepton can come from a $B \rightarrow D \rightarrow l$ cascade or from a $B^{0}$ which has mixed. Some $D^{*}$ can come from the virtual $W$ in the $B$-decay. These mistags reduce the observable amplitude of the oscillations.

Furthermore various background sources contribute to the sample: $D^{*}$ 's from $B^{ \pm}$decays which give unmixed events and $D^{*}$ s from $c \bar{c}$ initial states which give mixed sign correlations. The $c \bar{c}$ background contributes only at short decay lengths.

Trivial background arises from misidentified hadrons, decay leptons and combinatorial background of the $D^{*}$. In these cases there should be no significant charge correlation between the 'lepton' and the ' $D^{*}$ ' sides. Some of these backgrounds can be efficiently reduced by cuts on the lepton transverse momentum $\left(p_{t}\right)$ and the $D^{*}$ momentum.

The effects discussed above are demonstrated in figure 1 . The most important modification is due to the lepton mistag which reduces the amplitude. The charm and combinatorial backgrounds distort the distribution at short decay length. The characteristic turnover of the charge correlation is shifted due to the additional $D^{0}$ decay length, but not greatly affected by any background.

\section{$3 \quad$ Experimental setup}

The ALEPH detector has been described elsewhere [6]. In 1991 ALEPH was equipped with a high resolution vertex detector (VDET). This consists of two layers of double sided silicon microstrip detectors at radii of $6.3 \mathrm{~cm}$ and $10.8 \mathrm{~cm}$. The inner layer covers $85 \%$ of the solid angle, the outer layer $69 \%$. The spatial resolution of the $r-\phi$ and $z$ coordinates is $12 \mu \mathrm{m}$ at normal incidence [7]. Charged particles, once outside VDET, cross the inner tracking chamber (ITC) and the time projection chamber (TPC). The ITC is a cylindrical drift chamber with eight axial wire layers at radii between $16 \mathrm{~cm}$ and $26 \mathrm{~cm}$. The TPC provides up to 21 space points per track at radii between $40 \mathrm{~cm}$ and $171 \mathrm{~cm}$ and up to 330 measurements of the specific ionisation $(\mathrm{dE} / \mathrm{dx})$ of a track. Tracking is performed within a 

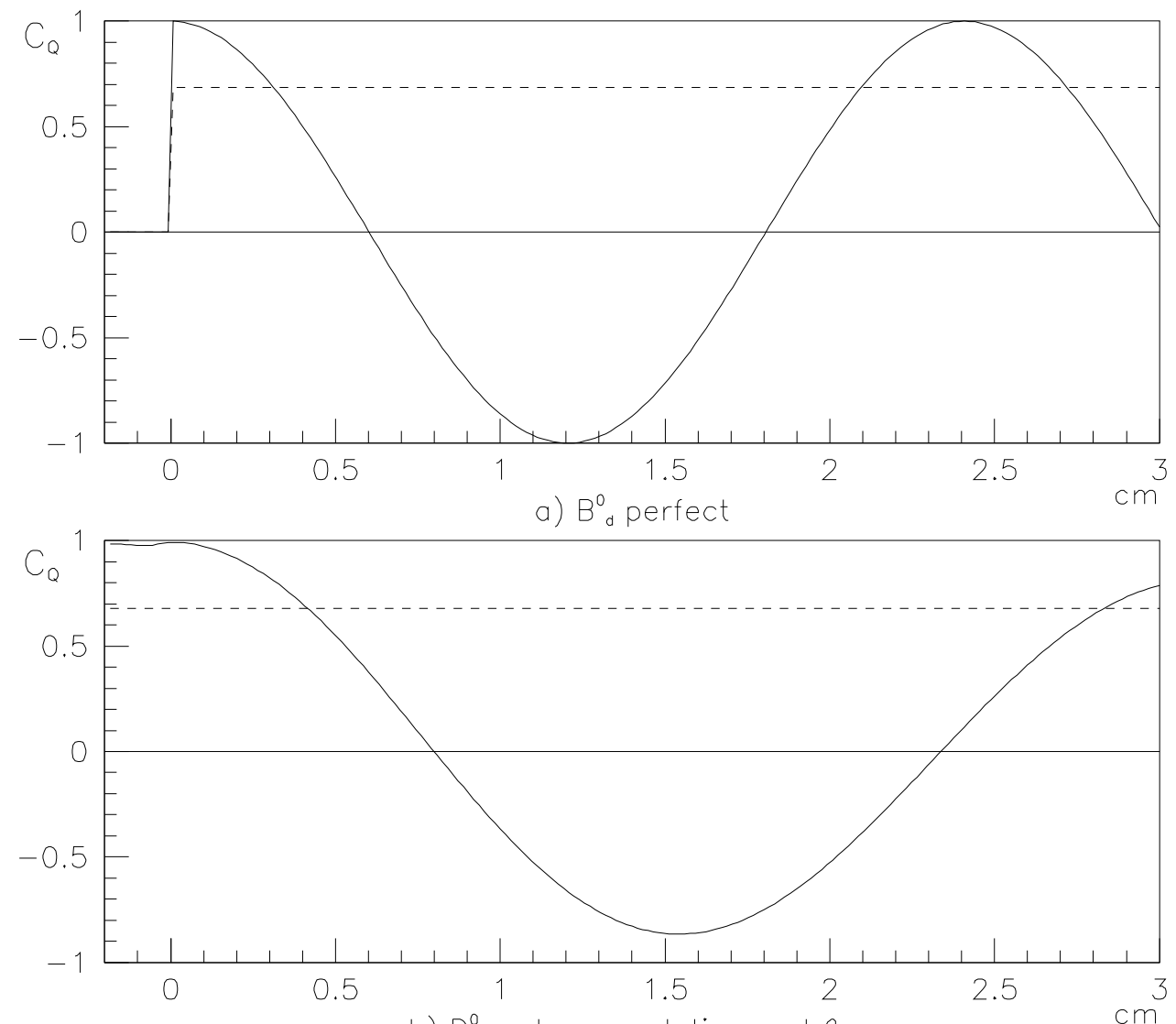

b) $D^{0}$ vertex, resolution and $\beta \gamma$

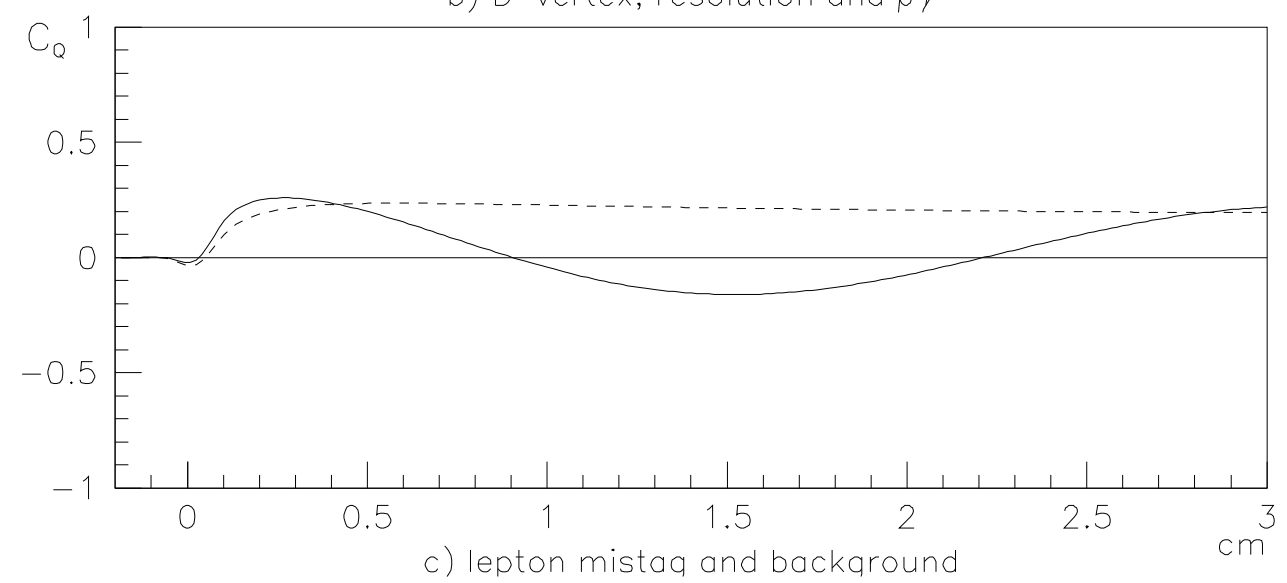

Figure 1: Charge correlation function plotted against decay length: a) for a pure $B_{d}^{0}$ sample with perfect $B_{d}^{0}$ vertex reconstruction and perfect charge tagging, fixed B-momentum; $\mathrm{b}$ ) using the $D^{0}$ vertex instead, convoluting with the vertex resolution and $B_{d}^{0}$ momentum distribution; c) including lepton mistag, adding charm, $B^{ \pm}$and combinatorial background. The dashed lines correspond to a hypothetical time independent mixing. 
$1.5 \mathrm{~T}$ magnetic field. The electromagnetic calorimeter (ECAL) is used in this analysis for electron identification and $\pi^{0}$ reconstruction. ECAL is a lead-proportional-tube calorimeter constructed in projective towers of $0.8^{0}$ by $0.8^{0}$ each. The hadron calorimeter (HCAL) with an average of $1.5 \mathrm{~m}$ of iron absorber and two layers of muon chambers provide $\mu$ identification.

\section{Event selection}

$D^{*}$ events are identified in the decay $D^{*+} \rightarrow D^{0} \pi^{+}$where a selection on the mass difference $m\left(D^{*}\right)-m\left(D^{0}\right)$ allows a powerful rejection of combinatorial background. The $D^{0}$ candidates are reconstructed in three decay channels:

$$
\begin{aligned}
& D^{0} \rightarrow K^{-} \pi^{+} \\
& D^{0} \rightarrow K^{-} \pi^{+} \pi^{0} \\
& D^{0} \rightarrow K^{-} \pi^{+} \pi^{-} \pi^{+} .
\end{aligned}
$$

The events are selected from about 900,000 events taken in the 1991 and 1992 run periods using the standard hadronic event selection described in [8]. Electrons are selected according to the shower shape in ECAL and $\mathrm{dE} / \mathrm{dx}$ information in the TPC. The muons selection is based on penetration in HCAL [9]. Jets are reconstructed from charged tracks and neutral energy in the calorimeters [10] and the lepton $p_{t}$ is calculated with the lepton removed from the jet. Leptons with a momentum above $3 \mathrm{GeV} / \mathrm{c}$ and a transverse momentum above $0.75 \mathrm{GeV} / \mathrm{c}$ are selected for this analysis. In $b$ events $77 \%$ of the leptons come from a primary $b$ decay, $14 \%$ from a $b \rightarrow c$ cascade, $2 \%$ from $b \rightarrow \tau$, the remainder are misidentified hadrons.

Tracks for $D^{*}$ reconstruction have to cross a virtual cylinder of $2 \mathrm{~cm}$ radius and $20 \mathrm{~cm}$ length centered at the nominal interaction point. Tracks are required to have at least four hits in the TPC and a $\chi^{2} / D O F$ of the helix fit of less than 4. Tracks which come from an identified $V^{0}$ or a $\gamma$ conversion are rejected.

In the $K \pi$ channel $D^{*}$ candidates are accepted if the $K \pi$ mass is between $\pm 20 \mathrm{MeV} / \mathrm{c}^{2}$ of the $D^{0}$ mass of $1.865 \mathrm{GeV} / \mathrm{c}^{2}$ and if the $D^{*}-D^{0}$ mass difference is within $144 \mathrm{MeV} / \mathrm{c}^{2}$ and $147 \mathrm{MeV} / \mathrm{c}^{2}$. The $D^{*}$ momentum has to be above $7 \mathrm{GeV} / \mathrm{c}$. In the two other channels the combinatorial background is much higher. Hence more severe selection requirements have to be applied. For the $K \pi \pi \pi$ channel the $\mathrm{dE} / \mathrm{dx}$ of the kaon and pions is required to be compatible with the respective hypothesis within two standard deviations. The $D^{0}$ mass window is $\pm 15 \mathrm{MeV} / \mathrm{c}^{2}$ and the $D^{*}$ momentum cut is increased to $10 \mathrm{GeV} / \mathrm{c}$.

The $\mathrm{dE} / \mathrm{dx}$ compatibility is also required in the $K \pi \pi^{0}$ channel. Neutral pions are reconstructed from $\gamma$ candidates measured in ECAL with $\left|m(\gamma \gamma)-m\left(\pi^{0}\right)\right|<50 \mathrm{MeV} / \mathrm{c}^{2}$ and $\mathrm{p}\left(\pi^{0}\right)>2.0 \mathrm{GeV} / \mathrm{c}$. The $D^{0}$ mass window is $66 \mathrm{MeV} / \mathrm{c}^{2}$ and the $D^{*}-D^{0}$ mass difference has to be within 143.4 and $148.6 \mathrm{MeV} / \mathrm{c}^{2}$. The $D^{*}$ momentum must be above $11 \mathrm{GeV} / \mathrm{c}$.

Finally the $D^{*}$ momentum is required to be less than $25 \mathrm{GeV} / \mathrm{c}$ in all samples. This removes a large fraction of $c \bar{c}$ events. At least two tracks must have VDET hits, to allow precise vertexing. If there are multiple candidates in the same events only the combination with the $D^{0}$ candidate closest to the nominal $D^{0}$ mass is kept. In the rare case that several candidates share the same $D^{0}$, the selection is made based on the $D^{*}-D^{0}$ mass difference.

The number of signal events and the combinatorial background are obtained from a fit 
to the $m\left(D^{*}\right)-m\left(D^{0}\right)$ mass spectra shown in figure 2. The signal is fitted with a gaussian, the background using a polynominal. The results are listed in table 1.

The vertex of the $D^{0}$ candidate is reconstructed in three dimensions. The primary vertex is reconstructed using all charged tracks as well as the known position and size of the interaction region [11]. The 'most probable decay length' of the $D^{0}$ as defined in [12] is then calculated.

\begin{tabular}{|c||c|c||c|c|}
\hline sample & $\begin{array}{c}\text { total } \\
\text { events }\end{array}$ & background & $\begin{array}{c}1>0 \mathrm{~cm} \\
\text { events }\end{array}$ & background \\
\hline $\mathrm{K} \pi$ & 105 & $18.0 \pm 2.0$ & 101 & $15.5 \pm 1.9$ \\
$\mathrm{~K} \pi \pi^{0}$ & 367 & $222.0 \pm 20.0$ & 297 & $178.0 \pm 18.0$ \\
$\mathrm{~K} \pi \pi \pi$ & 191 & $102.0 \pm 10.0$ & 169 & $91.0 \pm 9.0$ \\
\hline
\end{tabular}

Table 1: Signal and background from a fit to real data sidebands of the $D^{*}-D^{0}$ mass difference.

\section{Experimental result and extraction of the oscillation fre- quency}

The experimental result for the charge correlation function is shown in Figure 3. The data show the expected variation as function of the decay length. The oscillation frequency is obtained from a unbinned maximum likelihood fit to the decay length distributions of the like and unlike sign events. The fit functions of the $B_{d}^{0}$ contribution are derived from the time distributions (1) and (2) adding the effect of the $D^{0}$ decay. They are converted into decay length by convoluting them with the $B^{0}$ and $D^{0}$ momentum spectra taken from Monte Carlo simulations. Finally they are convoluted with the resolution function. This decay length resolution has been measured using data from track combinations in events which have been tagged as light quarks (uds) using an impact parameter method [11]. In $u d s$ events any vertex, after $V^{0}$ rejection, should coincide with the primary vertex and any deviation is a measure of the resolution. The typical resolutions are 230,350 , and $380 \mu \mathrm{m}$ for the $K \pi, K \pi \pi^{0}$, and $K \pi \pi \pi$ samples respectively.

The fit functions for the $B^{ \pm}, B_{s}^{0}$, and $c \bar{c}$ backgrounds are calculated following similar principles. The shape and charge correlation of the combinatorial background is obtained from the $D^{*}$ sidebands. The likelihood function for the unlike sign events can be written as:

$$
\begin{aligned}
\frac{1}{n} \frac{d n}{d l}(l) & =f_{B 0}\left[(1-A) \frac{1}{n} \frac{d n}{d l}(l, \Delta m)^{\text {mixed }}+A \frac{1}{n} \frac{d n}{d l}(l, \Delta m)^{\text {unmixed }}\right] \\
& +b k g\left(f_{B \pm}, f_{c \bar{c}}, f_{b k g}, f_{B s}, l\right)^{\text {unlike }}
\end{aligned}
$$

and for the like sign events:

$$
\begin{aligned}
\frac{1}{n} \frac{d n}{d l}(l) & =f_{B 0}\left[(1-A) \frac{1}{n} \frac{d n}{d l}(l, \Delta m)^{\text {unmixed }}+A \frac{1}{n} \frac{d n}{d l}(l, \Delta m)^{\text {mixed }}\right] \\
& +b k g\left(f_{B \pm}, f_{c \bar{c}}, f_{b k g}, f_{B s}, l\right)^{l i k e}
\end{aligned}
$$



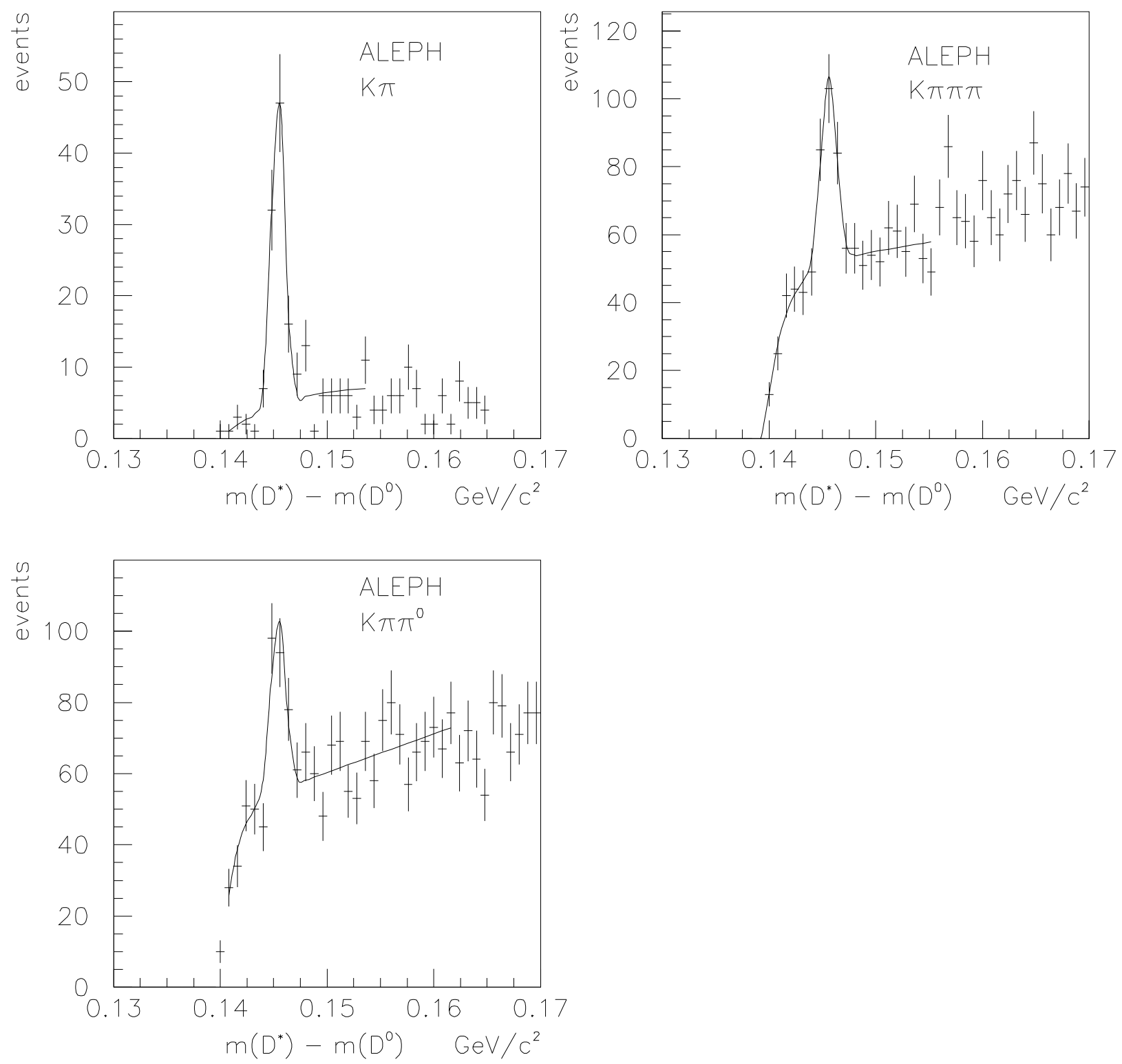

Figure 2: Mass difference $m\left(D^{*}\right)-m\left(D^{0}\right)$ for the samples $K \pi, K \pi \pi \pi$ and $K \pi \pi^{0}$ after lepton requirement. The solid line indicates the fit used for the determination of signal and background. 
$f_{B 0}$ is the fraction of $B_{d}^{0}$ events; the probability of a wrong sign association is given by $A$; 'bkg' denotes the contributions from $B^{ \pm}, B_{s}^{0}, c \bar{c}$, and combinatorial backgrounds. The fraction of events from $B^{ \pm},\left(f_{B+}\right)$, and $c \bar{c},\left(f_{c \bar{c}}\right)$, are free parameters of the fit. The combinatorial background is constrained to its expected value within its error. Furthermore the sign mistag fraction $A$ is allowed to vary freely. Only events with positive decay length are used in the fit. All three subsamples are fitted simultaneously. The result of the fit is:

$$
\begin{aligned}
\Delta m & =0.52_{-0.11}^{+0.10} \hbar / \mathrm{ps} \\
A & =0.23 \pm 0.04 \\
f_{c \bar{c}} & =0.06 \pm 0.04 \\
f_{B+} & =0.00_{-0.00}^{+0.22} .
\end{aligned}
$$

The correlation between $\Delta m$ and the other parameters are small ( 0.26 for $f_{B+}$ and $<0.06$ for $A$ and $f_{c \bar{c}}$ ). The charge correlation function calculated from the fit is shown in figure 3 . If the fit is repeated using a time independent model, the negative log likelihood increases by 5.2 , corresponding to 3.2 standard deviations.

The results on $B^{ \pm}$fraction, charm fraction, and mistag can be compared to expectations providing a consistency check of the fit:

- In a simple spectator model one would expect that $B_{d}^{0} \rightarrow D^{*-} X$ dominates over $B^{+} \rightarrow D^{*-} X$, hence most of the $D^{*}$ come from $B^{0}$. This is indeed observed in semileptonic decays [12] where the fraction of $D^{*}$ from $B^{ \pm}$has been measured to be $f_{B+}=0.16_{-0.16}^{+0.10}$. This should be a reasonable assumption for the inclusive $D^{*}$ sample. The fitted value of $0.00_{-0.00}^{+0.22}$ is in agreement with these expectations and confirms that the $B^{ \pm}$contribution is small.

- Using the Monte Carlo selection efficiencies a charm fraction of $0.09 \pm 0.02$ is expected. The fit result of $0.06 \pm 0.04$ is in good agreement with the expectations.

- The mistag probability of leptons from $b$-decays can be extracted from Monte Carlo: $A=0.25 \pm 0.01$. An additional contribution can arise due to $D^{*}$ from the virtual W, like $B^{0} \rightarrow D^{*+} K^{0} D^{*-}$ or $B_{d}^{0} \rightarrow D^{*+} D^{-}$. These decays are either phase space or Cabibbo suppressed. They could add about $0.03 \pm 0.03$ to $A$. $A$ agrees very well with the expected value from lepton mistag alone and does not leave much room for a large contribution of $D^{*}$ from the $W$.

\section{Systematic errors}

The following systematic errors have been studied:

- $B^{ \pm}$charge correlation: Although the fraction of wrong sign $D^{*}$ from the virtual $\mathrm{W}$ is small for the $B^{0}$, its relative magnitude may be more important in the case of $B^{ \pm}$. Hence the mistag fraction of $B^{ \pm}$, nominally 0.38 , is varied in the range of 0.25 to 0.50 resulting in an error of $\pm 0.02 \hbar / p s$ on $\Delta m$. 


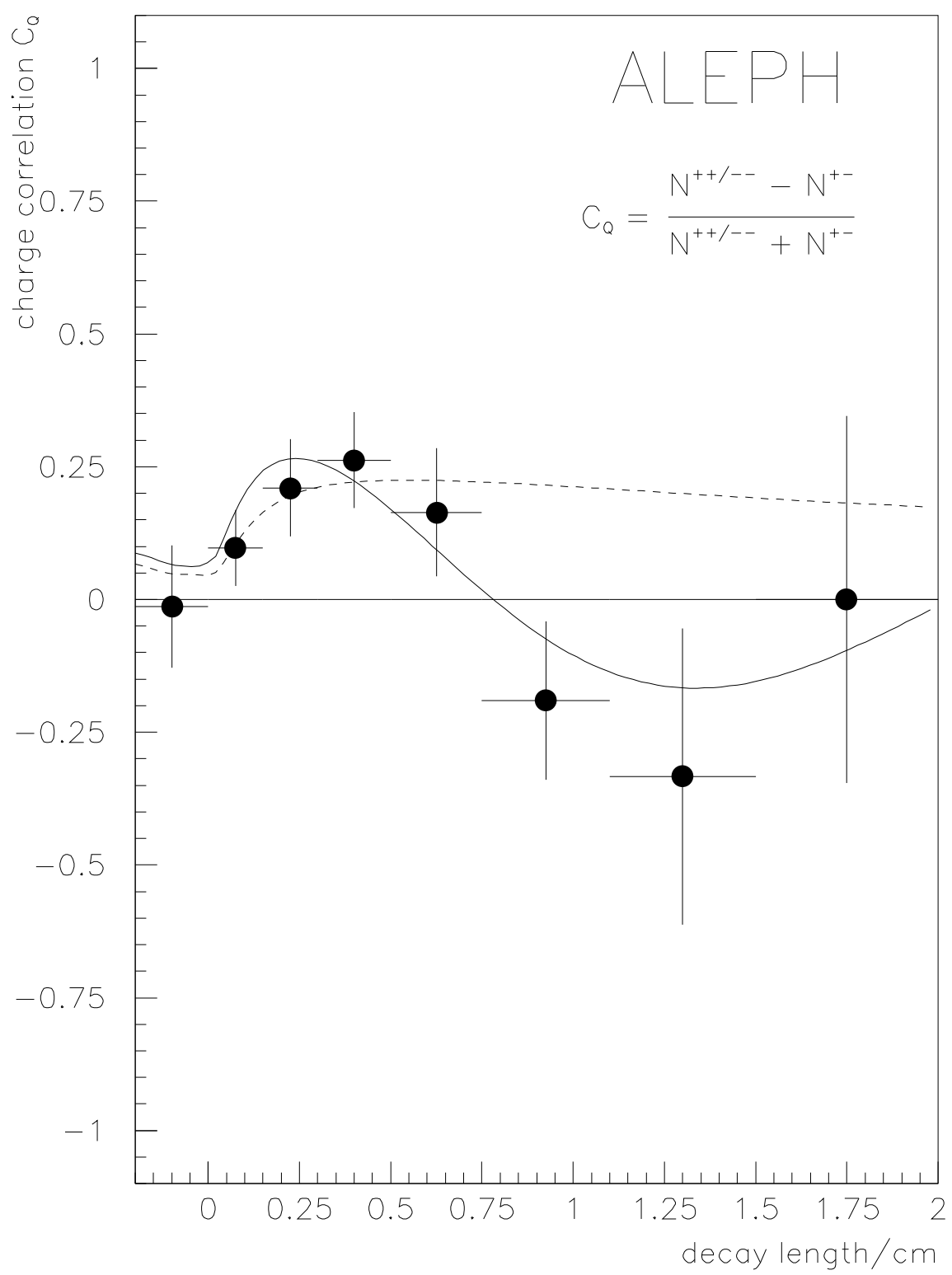

Figure 3: Experimental result of the charge correlation function $C_{Q}$. The result of the fit allowing for a time dependent oscillation is indicated as solid line. The time independent alternative is shown as dashed line. 
- Lifetimes: As the fit measures the oscillation frequency $\Delta m$, the uncertainty of the $B_{d}^{0}$ lifetime gives only a small error. Combining exclusive lifetime measurements by ALEPH [12] and DELPHI [13] and OPAL [14] one obtains:

$$
\tau_{B_{d}^{0}}=1.44 \pm 0.15 \mathrm{ps} ; \quad \tau_{B^{ \pm}}=1.42 \pm 0.17 \mathrm{ps},
$$

which are used for estimating the systematic error. Changing the $B^{0}$ lifetime by its error changes the result on $\Delta m$ by ${ }_{-0.01}^{+0.03} \hbar / \mathrm{ps}$, whilst $\frac{\Delta m}{\Gamma}$ varies by ${ }_{-0.05}^{+0.07}$. The errors due to the $B^{ \pm}$and $D^{0}$ lifetimes are listed in table 2 .

- Fragmentation functions: The $b$ fragmentation function affects the $B_{d}^{0}$ momentum spectrum, which enters directly in the measured value of $\Delta m$. Using $\bar{x}_{b}=0.712 \pm 0.012$ from reference [15] results in an error on $\Delta m$ of $\pm 0.01 \hbar / p s$.

- $D^{0}$ moment um spectrum: The shape of the momentum spectrum of $D^{0}$ was compared between data and Monte Carlo. The difference in the average momentum is less than $5 \%$. Attributing this difference to uncertainties in the $B^{0}$ decay kinematics leads to an error of $\pm 0.01 \hbar /$ ps on $\Delta m$.

- Resolution function: Various parameterizations of the resolution function have been used to estimate the systematics, giving $\pm 0.01 \mathrm{~h} / \mathrm{ps}$.

- Background subtraction: The systematic error due to the combinatorial background is dominated by the uncertainty of its charge correlation. This introduces an error of ${ }_{-0.003}^{+0.004} \hbar / \mathrm{ps}$ on $\Delta m$.

- $B_{s}^{0}$ contribution: The amount of $D^{*}$ from $B_{s}^{0}$ is expected to be about $5 \%$ of the $D^{*} \mathrm{~s}$ from $B_{d}^{0}$ and is taken into account in the fit. Because of the small contribution the fit is not sensitive to the actual value of $\Delta m$ for $B_{s}^{0}$. The error from this background is negligible.

The systematic errors are summarized in table 2. The total systematic error on $\Delta m$ is :

$$
\sigma(\Delta m)_{s y s}={ }_{-0.03}^{+0.04} \hbar / \mathrm{ps} .
$$

\section{Conclusions}

Using $D^{*}$-lepton correlations the characteristic time dependence of $B_{d}^{0} \bar{B}_{d}^{0}$ oscillations has been observed. A model without time dependence of the transition probability is disfavoured by 3.2 standard deviations. The measured mass difference $\Delta m$ is:

$$
\Delta m=0.52{ }_{-0.11}^{+0.10}(\text { stat }){ }_{-0.03}^{+0.04}(\text { sys }) \hbar / \mathrm{ps},
$$

or, converting into $\mathrm{eV} / \mathrm{c}^{2}$ :

$$
\Delta m=\left[3.44_{-0.70}^{+0.65}(\text { stat }){ }_{-0.20}^{+0.26}(\text { sys })\right] 10^{-4} \mathrm{eV} / \mathrm{c}^{2} .
$$




\begin{tabular}{|c||c|c|}
\hline quantity & input and error & resulting systematic error \\
\hline \hline$B^{ \pm}$charge correlation & $0.38 \pm 0.13$ & $\pm 0.02 \hbar / \mathrm{ps}$ \\
$B_{d}^{0}$ lifetime & $1.44 \pm 0.15 \mathrm{ps}$ & ${ }_{-0.01}^{+0.03} \hbar / \mathrm{ps}$ \\
$B^{ \pm}$lifetime & $1.42 \pm 0.17 \mathrm{ps}$ & ${ }_{-0.01}^{+0.00} \hbar / \mathrm{ps}$ \\
$D^{0}$ lifetime & $0.42 \pm 0.008 \mathrm{ps}$ & $\pm 0.004 \hbar / \mathrm{ps}$ \\
$b$ fragmentation $x_{b}$ & $0.712 \pm 0.012$ & $\pm 0.01 \hbar / \mathrm{ps}$ \\
$D^{0}$ momentum & $13.4 \pm 0.7 \mathrm{GeV} / \mathrm{c}$ & $\pm 0.01 \hbar / \mathrm{ps}$ \\
resolution & - & $\pm 0.01 \hbar / \mathrm{ps}$ \\
backg. charge correlation & - & ${ }_{-0.003}^{+0.004} \hbar / \mathrm{ps}$ \\
\hline total & & ${ }_{-0.03}^{+0.04} \hbar / \mathrm{ps}$ \\
\hline
\end{tabular}

Table 2: Systematic errors on $\Delta m$.

This is a direct measurement of the mass difference. Using $\tau_{B_{d}^{0}}=1.44 \pm 0.15 \mathrm{ps}$, this corresponds to a value of $\frac{\Delta m}{\Gamma}$ of:

$$
\frac{\Delta m}{\Gamma}=0.75_{-0.14}^{+0.15}(\text { stat })_{-0.05}^{+0.08}(\text { sys }) \text {. }
$$

The extra error due to the $B_{d}^{0}$ lifetime is included in the systematic error. This can be compared with the values extracted from the $\chi_{d}$ measurements of ARGUS: [3]: $\frac{\Delta m}{\Gamma}=0.72 \pm 0.15$, and CLEO [4]: $\frac{\Delta m}{\Gamma}=0.65 \pm 0.10$.

\section{Acknowledgements}

We would like to thank our colleagues of the accelerator division for the excellent performance of LEP. Thanks are also due to the many engineering and technical personnel at CERN and at the home institutes for their contributions towards the success of ALEPH. Those of us not from member states wish to thank CERN for its hospitality.

\section{References}

[1] A.J. Buras, W. Slominski and H. Steger, Nucl. Phys. B245 (1984) 369

[2] UA1 Collaboration, C. Albajar et al., Phys. Lett. B186 (1987) 247

[3] ARGUS Collaboration, H. Albrecht et al., Phys. Lett. B192 (1987) 245 ARGUS Collaboration, H. Albrecht et al.,Z. Phys. C55 (1992) 363

[4] CLEO Collaboration, M. Artuso et al., Phys. Rev. Lett. 62 (1989) 2233, H. Kroha, Modern Physics Letters A, Vol. 8, No 10 (1993) 869

[5] UA1 Collaboration, C. Albajar et al., Phys. Lett. B262 (1991) 171 ALEPH Collaboration, D. Decamp et al., Phys. Lett. B258 (1991) 236 
OPAL Collaboration, P.D. Acton et al., Phys. Lett. B276 (1992) 379

ALEPH Collaboration, D. Buskulic et al., Phys. Lett B284 (1992) 177

L3 Collaboration, B. Adeva et al., Phys. Lett. B288 (1992) 395

DELPHI Collaboration, P. Abreu et al., Phys. Lett. B301 (1993) 145

[6] ALEPH Collaboration, D. Decamp et al., Nucl. Inst. and Meth. A294 (1990) 121

[7] G. Batignani et al., Recent results and running experience of the new ALEPH vertex detector, Conf. Record of the 1991 IEEE Nuclear Science Symp., (November 1991, Santa Fe, NM, USA), Vol. 1, 438

[8] ALEPH Collaboration, D. Decamp et al., Z. Phys, C53 (1992) 1.

[9] ALEPH Collaboration, D. Decamp et al, Phys. Lett B263 (1991) 325

[10] JADE Collaboration, C. Kleinwort et al., Z. Phys. C42 (1989) 7

[11] ALEPH Collaboration, D. Buskulic et al., A precise measurement of the ratio $\Gamma_{Z \rightarrow b \bar{b}} / \Gamma_{Z \rightarrow \text { hadrons }}$; to be published

[12] ALEPH Collaboration, D. Buskulic et al., Measurement of the $B^{0}$ and $B^{-}$Meson Lifetimes, CERN PPE/93-42, submitted to Physics Letters B

[13] DELPHI Collaboration, P. Abreu et. al., A Measurement of the B meson Production and Lifetime using $\mathrm{Dl}^{-}$Events in $Z^{0}$ decays; CERN-PPE/92-174 (1992), submitted to Zeit. Phys. C

[14] OPAL Collaboration, P.D. Acton et. al., Measurement of the $B^{0}$ and $B^{+}$lifetimes CERN-PPE/93-33 (1993)

[15] ALEPH Collaboration, D. Buskulic et al., Heavy Flavour Quark Production and Decay Using Prompt Leptons in the ALEPH Detector, in preparation 